\title{
FACTORIZATION OF POLYNOMIALS OVER BANACH ALGEBRAS(1)
}

\author{
BY \\ JOHN A. LINDBERG, JR.
}

Introduction. A Banach algebra in this paper will be understood to mean a commutative, semi-simple Banach algebra with multiplicative unit $e$. By the carrier space $\Phi_{A}$ of the Banach algebra $A$, we mean the space of multiplicative linear functionals on $A$ to $C$, the complex numbers, and it is to be endowed with the usual weak* topology (cf. [6]). For $a \in A$, $\hat{a}$ denotes the Gelfand transform of $a$ defined on $\Phi_{A}$ and $\hat{A}$ will denote the collection of such functions. We set the following notation. $x$ will be used to denote an indeterminate over $A$ as well as over $\hat{A}$ and $C$. If $\alpha(x)=\sum_{i=0}^{n} \alpha_{i} x^{i}$ is a polynomial over $A$, let $\hat{\alpha}(x)$ and $\alpha_{h}(x)$ denote, respectively, $\sum_{i=0} \hat{\alpha}_{i} x^{i}$ and $\sum_{i=0}^{n} \hat{\alpha}_{i}(h) x^{i}, h \in \Phi_{A}$.

The set $Z(\alpha(x), A)=\left\{(h, \lambda) \in \Phi_{A} \times C: \alpha_{h}(x) \neq 0\right.$ and $\left.\alpha_{h}(\lambda)=0\right\}, \alpha(x) \in A[x]$, plays an important role in the present paper. $Z(\alpha(x), A)$ is topologized with the relative product topology from $\Phi_{A} \times C$. The mapping $\pi$ is defined by $\pi(h, \lambda)=h,(h, \lambda) \in Z(\alpha(x), A)$. The multiplicity function $M$ of $\alpha(x)$ is defined as follows: for $(h, \lambda) \in Z(\alpha(x), A), M(h, \lambda)$ is equal to the multiplicity of $\lambda$ as a root of $\alpha_{h}(x)=0$.

In $\$ 1$ we introduce the concept of $M$-neighborhood of a point in $Z(\alpha(x), A)$. We say that $W \subset Z(\alpha(x), A)$ is a $M$-neighborhood of $\left(h_{0}, \lambda_{0}\right) \in Z(\alpha(x), A)$ if $W$ is a neighborhood in $Z(\alpha(x), A)$ of $\left(h_{0}, \lambda_{0}\right)$ and if, for each $h \in \pi(W), M\left(h_{0}, \lambda_{0}\right)$ is equal to the sum of the values of $M$ at the points $(h, \lambda)$ in $\pi^{-1}(h) \cap W$. Proposition 1.1 states that $M$-neighborhoods exist and that they form a base for the neighborhood system at each point of $Z(\alpha(x), A)$. The remainder of this section contains most of the topological lemmas needed for our work on factorization. Particular attention is paid to the case where $Z(\alpha(x), A)$ contains a compact open subset $K\left(\pi(K)=\Phi_{A}\right)$ on which $M$ is constant. When this condition obtains, $K$ and $\Phi_{A}$ decompose topologically and this decomposition in turn forces $\alpha(x)$ to factor.

The main factorization theorem (2.1) says that if $\alpha(x) \in A[x]$ and if $K\left(\pi(K)=\Phi_{A}\right)$ is a compact open subset of $\mathrm{Z}(\alpha(x), A)$, then there exists a monic polynomial $\beta(x) \in A[x]$ such that $\beta(x)$ is a factor of $\alpha(x), Z(\beta(x), A)=K$ and $\mathrm{Z}(\alpha(x) / \beta(x), A)=\mathrm{Z}(\alpha(x), A) \sim K$. A more detailed description of the factorization of monic polynomials follows. For example, it is shown that if $A$ is indecomposable

Received by the editors February 5, 1962 and, in revised form, April 3, 1963.

(1) This paper is based on portions of the author's Doctoral dissertation which was prepared under the guidance of Professor B. R. Gelbaum, University of Minnesota. 
and if $M$ is locally constant on $Z(\alpha(x), A)$, then the factorization of $\alpha(x)$ into irreducible factors is unique (to within units).

$\$ 3$ deals with the problem of finding zeros of a given polynomial over $A$ in $A$ and in extensions of $A$ which are also Banach algebras. Theorem 3.2 extends, for semi-simple Banach algebras, a theorem (4.3 [1]) of Arens and Calderón. Our theorem states that if $\alpha(x) \in A[x], f \in C\left(\Phi_{A}\right)$ such that $\hat{\alpha}(f)=0$ and $M(\cdot, f(\cdot))$ is locally constant on $\Phi_{A}$, then $f \in \hat{A}$.

1. Some topological results. Let $A$ be a Banach algebra and

$$
\alpha(x)=\sum_{i=0}^{n} \alpha_{i} x^{i} \in A[x] .
$$

(The results of this section do not depend on the semi-simplicity of $A$.) The topology in $\Phi_{A}$ is to be the usual weak* topology. Then the topology in $Z(\alpha(x), A)$ induced by the product topology in $\Phi_{A} \times C$ is precisely the weakest topology which renders the functions $\sum_{i=0}^{m} \hat{a}_{i} f^{i}, a_{i} \in A$, continuous on $Z(\alpha(x), A)$, where $f(h, \lambda) \equiv \lambda$ for $(h, \lambda) \in Z(\alpha(x), A)$. From its definition, it is easily seen that $\pi$ is continuous with respect to the prescribed topologies and it follows from Proposition 1.1 that $\pi$ is also an open mapping. $\pi(Z(\alpha(x), A))$ need not be all of $\Phi_{A}$; however, it is an open subset of $\Phi_{A}$.

Definition. A neighborhood $W$ in $Z(\alpha(x), 4)$ of $\left(h_{0}, \lambda_{0}\right)$ is called a $M$-neighborhood of $\left(h_{0}, \lambda_{0}\right)$ if, for each $h \in \pi(W)$,

$$
M\left(h_{0}, \lambda_{0}\right)=\sum_{(h, \lambda) \in \pi^{-1}(h) \cap W} M(h, \lambda),
$$

that is, $M\left(h_{0}, \lambda_{0}\right)$ is equal to the sum of the values of $M$ at the points in $\pi^{-1}(h) \cap W$.

It follows from the above definition that if $W$ is a $M$-neighborhood of $(h, \lambda)$ and if $V$ is a neighborhood in $\Phi_{A}$ of $h$, then $\pi^{-1}(V) \cap W$ is also a $M$-neighborhood of $(h, \lambda)$.

Proposition 1.1. Let $\alpha(x)=\sum_{i=0}^{n} \alpha_{i} x^{i} \in A[x]$ and $\left(h_{0}, \lambda_{0}\right) \in Z(\alpha(x), A)$. If $W$ is a neighborhood in $Z(\alpha(x), A)$ of $\left(h_{0}, \lambda_{0}\right)$, then there exists a M-neighborhood $W_{0}$ of $\left(h_{0}, \lambda_{0}\right)$ which is contained in $W$.

Proof. It suffices to prove the proposition for the case where $W$ is of the form $\left(V \times\left\{\lambda \in C:\left|\lambda-\lambda_{0}\right|<\varepsilon\right\}\right) \cap Z(\alpha(x), A)$, where $\varepsilon>0$ and $V$ is a neighborhood in $\Phi_{A}$ of $h_{0}$. Consider the polynomial $p\left(z_{0}, z_{1}, \cdots, z_{n}, w\right)$ $=z_{0}+z_{1} w+\cdots+z_{n} w^{n}$. Then $\lambda_{0}$ is a root of $p\left(\hat{Q}_{0}\left(h_{0}\right), \hat{Q}_{1}\left(h_{0}\right), \cdots, \hat{Q}_{n}\left(h_{0}\right), w\right)=0$. Suppose that $\lambda_{0}$ is a root of multiplicity $m$. From the Weierstrass preparation theorem, it follows that there exists $s>0$ and $t>0$ such that if $\left|z_{i}-\hat{Q}_{i}\left(h_{0}\right)\right|<t$, $i=0,1, \cdots, n$, then $p\left(z_{0}, z_{1}, \cdots, z_{n}, w\right)=0$ possesses exactly $m$ roots (multiplicities counted) in $\left\{w \in C:\left|w-\lambda_{0}\right|<s\right\}$ (cf. [5]). Furthermore, $s$ can be so chosen that it is less than $\varepsilon$. Now, $V_{0}=\left\{h \in \Phi_{A}:\left|\hat{\alpha}_{i}(h)-\hat{\alpha}_{i}\left(h_{0}\right)\right|<t, i=0,1, \cdots, n\right\}$ is a neighborhood in $\Phi_{A}$ of $h_{0}$ so that $V \cap V_{0}$ is also a neighborhood in $\Phi_{A}$ of $h_{0}$. Let $W_{0}$ 
denote the neighborhood $\left(\left(V \cap V_{0}\right) \times\left\{\lambda \in C:\left|\lambda-\lambda_{0}\right|<s\right\}\right) \cap Z(\alpha(x), A)$. Then $W_{0} \subset W$ and $W_{0}$ is a $M$-neighborhood of $\left(h_{0}, \lambda_{0}\right)$.

Q.E.D.

The above proposition tells us that the $M$-neighborhoods of a point in $Z(\alpha(x), A)$ form a base for the neighborhood system of that point.

From the construction in the proof of the proposition we have the following important result:

1.2. $\pi$ is an open mapping.

This can be seen in the following way. Let $W$ be an open subset of $Z(\alpha(x), A)$ and let $(h, \lambda) \in W$. Then let $W_{0}$ be the $M$-neighborhood of $(h, \lambda)$ contained in $W$ which was constructed above. It has the property that $\pi\left(W_{0}\right)$ is a neighborhood in $\Phi_{A}$ of $h$. Thus it follows that $\pi(W)$ is open and that $\pi$ must be an open mapping.

Since the topology in $Z(\alpha(x), A)$ is Hausdorff, we can conclude from the proposition that any two distinct points of $Z(\alpha(x), A)$ possess disjoint $M$-neighborhoods. If the points have the same first coordinate, then $M$-neighborhoods can be found which have the same image under $\pi$. For if $W_{1}$ and $W_{2}$ are the disjoint $M$-neighborhoods of these points, then $V=\pi\left(W_{1}\right) \cap \pi\left(W_{2}\right)$ is a neighborhood so that $\pi^{-1}(V) \cap W_{1}$ and $\pi^{-1}(V) \cap W_{2}$ are the desired $M$-neighborhoods.

In the next section we concern ourselves with subsets $K$ of $Z(\alpha(x), A)$ which are compact and open, and such that $\pi(K)=\Phi_{A}$. The next proposition shows such sets behave, with respect to $\Phi_{A}$, in a manner which is reminiscent of covering spaces (cf. [3]). If $h \in \Phi_{A}$, let $r(h)$ denote the cardinality of $\pi^{-1}(h) \cap K$ and let $\left(h, \lambda_{1}\right),\left(h, \lambda_{2}\right), \cdots,\left(h, \lambda_{r(h)}\right)$ denote the distinct points in $\pi^{-1}(h) \cap K$.

Proposition 1.3. Let $\alpha(x)=\sum_{i=0}^{n} \alpha_{i} x^{i} \in A[x]$ and $K$ be a compact open subset of $\mathrm{Z}(\alpha(x), A)$ such that $\pi(K)=\Phi_{A}$. Then for each $h \in \Phi_{A}$, there are disjoint $M$-neighborhoods $V_{k}(h)$ of the points $\left(h, \lambda_{k}\right)$ and positive numbers (depending on $h) t$ and $s_{k}, k=1,2, \cdots, r(h)$ such that

(i) for each $k, V_{k}(h)$ is contained in $\left\{\left(h^{\prime}, \lambda^{\prime}\right) \in K:\left|\hat{\alpha}_{j}\left(h^{\prime}\right)-\hat{\alpha}_{j}(h)\right|<t\right.$, $j=0,1, \cdots, n$ and $\left.\left|\lambda-\lambda_{k}\right|<s_{k}\right\}$,

(ii) for each $k, \pi\left(V_{k}(h)\right)=\pi\left(V\left(h_{1}\right)\right)$, and

(iii) $\pi^{-1}\left(\pi\left(V_{1}\right)\right) \cap K=\bigcup_{k=1}^{r(h)} V_{k}(h)$.

Furthermore, if $U(h)=\left\{\left(z_{0}, z_{1}, \cdots, z_{n}\right) \in C^{n+1}:\left|z_{j}-\hat{\alpha}_{j}(h)\right|<t, j=0,1, \cdots, n\right\}$, then

(iv) $p\left(z_{0}, z_{1}, \cdots, z_{n}, w\right)=\sum_{i=0} z_{i} w^{l}$ has exactly $M\left(h, \lambda_{k}\right)$ roots (each distinct root repeated according to its multiplicity) in $\left\{\lambda \in C:\left|\lambda-\lambda_{k}\right|<s_{k}\right\}$ if $\left(z_{0}, z_{1}, \cdots, z_{n}\right) \in U(h)$.

Proof. Let $W_{1}, \cdots, W_{r(h)}$ be disjoint neighborhoods of the points $\left(h, \lambda_{1}\right), \cdots,\left(h, \lambda_{r(h)}\right)$. In view of Proposition 1.1 (and its proof) and the fact that $K$ is open, we may and do assume that the $W_{k}$ 's are $M$-neighborhcods satisfying (i) for an appropriate choice of $t>0$ and $s_{k}>0, k=1,2, \cdots, r(h)$, and such that (iv) is satisfied for this choice. As seen earlier, the openness of $\pi$ allows us to assume that 
$\pi\left(W_{k}\right)=\pi\left(W_{1}\right), k=1,2, \cdots, r(h)$. Now let $W=\pi^{-1}\left(\pi\left(W_{1}\right)\right) \cap K \sim \bigcup_{k=1}^{r(h)} W_{k}$ and consider the closure $\bar{W}$ of $W$. Since $K$ is compact, $W$ is compact and $\pi(\bar{W})$ is a compact subset of $\Phi_{A}$; hence, $\pi(\bar{W})$ is a closed subset of $\Phi_{A}$. Since $\left(h, \lambda_{k}\right) \notin \bar{W}$, for each $k, h \notin \pi(\bar{W})$ so that there is a neighborhood $V$ in $\Phi_{A}$ of $h$ such that $\pi(W) \cap V=\varnothing$; thus, $\pi^{-1}(V) \cap W=\varnothing$. If we take $V_{k}=W_{k} \cap \pi^{-1}(V)$, then the above properties are satisfied with $t$ and the $s_{k}$ 's chosen as above.

Q.E.D.

By $M$ locally constant ( $\pi$ a local homeomorphism) at $(h, \lambda) \in Z(\alpha(x), A)$, we mean that there exists a neighborhood $W$ in $Z(\alpha(x), A)$ of $(h, \lambda)$ on which $M$ is constant $(\pi \mid W: W \rightarrow \pi(W)$ is a homeomorphism).

LEMMA 1.4. Let $\alpha(x) \in A[x]$. The following are equivalent:

(i) $M$ is locally constant at $(h, \lambda) \in Z(\alpha(x), A)$,

(ii) $\pi$ is a local homeomorphism at $(h, \lambda)$, and

(iii) there exists a neighborhood $V$ in $\Phi_{A}$ of $h$ and a function $f \in C(V)$ such that $\alpha_{h^{\prime}}\left(f\left(h^{\prime}\right)\right)=0$ for each $h^{\prime} \in V, M(\cdot, f(\cdot))$ is constant on $V$ and $f(h)=\lambda$.

Proof. We show (iii) $\Rightarrow$ (ii) $\Rightarrow$ (i) $\Rightarrow$ (iii).

(iii) $\Rightarrow$ (ii). Let $W$ be a $M$-neighborhood of $(h, \lambda)$ and let $V_{0}$ be a neighborhood in $\Phi_{A}$ of $h$ such that $V_{0} \subset V$ and $h^{\prime} \in V_{0}$ implies that $\left(h^{\prime}, f\left(h^{\prime}\right)\right) \in W$. Then $\pi^{-1}\left(V_{0}\right) \cap W$ is a $M$-neighborhood of $(h, \lambda)$ and hence we assume that $W=W \cap \pi^{-1}\left(V_{0}\right)$. Now, if $\pi \mid W$ is not a homeomorphism, it must be the case that there is a point $\left(h^{\prime}, \lambda^{\prime}\right) \in W$ such that $\lambda^{\prime} \neq f\left(h^{\prime}\right)$ since $\pi$ is an open mapping. But this means that $M\left(h^{\prime}, f\left(h^{\prime}\right)\right)<M\left(h^{\prime}, f\left(h^{\prime}\right)\right)+M\left(h^{\prime}, \lambda^{\prime}\right) \leqq M(h, f(h))$. This is a contradiction since $M(\cdot, f(\cdot))$ is constant on $V$ and so it follows that $\pi \mid W$ must be a homeomorphism.

(ii) $\Rightarrow$ (i). Suppose that $W$ is a neighborhood in $Z(\alpha(x), A)$ of $(h, \lambda)$ such that $\pi \mid W$ is a homeomorphism. It may be assumed that $W$ is a $M$-neighborhood since $W$ contains such a neighborhood. Now, $M$ must be constant on $W$. For if it is not, then there are two points $\left(h^{\prime}, \lambda^{\prime}\right)$ and $\left(h^{\prime}, \lambda^{\prime \prime}\right)$ in $W, \lambda^{\prime} \neq \lambda^{\prime \prime}$. But this is a contradiction so that $M$ is constant on $W$.

(i) $\Rightarrow$ (iii). Let $W$ be a neighborhood in $Z(\alpha(x), A)$ of $(h, \lambda)$ on which $M$ is constant. We assume that $W$ is a $M$-neighborhood of $(h, \lambda)$. Thus, the cardinality of $\pi^{-1}\left(h^{\prime}\right) \cap W$ is always equal to one if $h^{\prime} \in \pi(W)$. This means that $\pi \mid W$ is a homeomorphism so that if $f$ is defined to be the function on $V=\pi(W)$ such that $\left(h^{\prime}, f\left(h^{\prime}\right)\right) \in W, h^{\prime} \in V$, then $f$ is continuous on $V$. Furthermore, $\alpha_{h^{\prime}}\left(f\left(h^{\prime}\right)\right)=0$, $h^{\prime} \in V, f(h)=\lambda$ and $M(\cdot, f(\cdot))$ is constant on $V$.

Q.E.D.

If $K$ is a compact open subset of $Z(\alpha(x), A)$ such that $\pi(K)=\Phi_{A}$, then we define $m_{K}(h)$ to be the sum of the values of $M$ at the points of $\pi^{-1}(h) \cap K$. (If we are dealing in any given situation with only one such set $K$, we will simply denote $m_{K}$ by $m$.)

LeMMA 1.5. If $K$ is a compact open subset of $Z(\alpha(x), A)$ such that $\pi(K)=\Phi_{A}$, then there exists disjoint open subsets $X_{i}, i=1,2, \cdots, s$, of $\Phi_{A}$ such that $\bigcup_{i=1}^{s} X_{i}=\Phi_{A}$ and the function $m(\cdot)$ is constant on each $X_{i}$. 
Proof. For each $h \in \Phi_{A}$, let $\left(h, \lambda_{1}(h)\right), \cdots,\left(h, \lambda_{r(h)}(h)\right)$ denote the points in $\pi^{-1}(h) \cap K$ and let $X_{i}=\left\{h \in \Phi_{A}: m(h)=i\right\}$. Then the $X_{i}$ are open sets in $\Phi_{A}$. For if $h \in X_{i}$, for some $i$, then let $V_{k}, k=1,2, \cdots, r(h)$, be the neighborhoods of the $\left(h, \lambda_{k}\right)$ 's which were given in Proposition 1.3. Then $h^{\prime} \in \pi\left(V_{1}\right)$ implies that

$$
\begin{aligned}
m\left(h^{\prime}\right) & =\sum_{i=1}^{r\left(h^{\prime}\right)} M\left(h^{\prime}, \lambda\left(h^{\prime}\right)\right)=\sum_{i=1}^{r(h)}\left(\sum_{\left(h^{\prime}, \lambda^{\prime}\right) \in \pi^{-1}\left(h^{\prime}\right) \cap V_{i}} M\left(h^{\prime}, \lambda^{\prime}\right)\right) \\
& =\sum_{i=1}^{r(h)} M\left(h, \lambda_{i}(h)\right)=m(h)
\end{aligned}
$$

so that $h^{\prime} \in X_{i}$ and hence $\pi\left(V_{1}\right) \subset X_{i}$. Thus, each $X_{i}$ is open in $\Phi_{A}$. Clearly, the $X_{i}$ 's are disjoint.

Q.E.D.

We now turn our attention to the case where $\alpha(x)$ is a monic polynomial. In this case, $Z(\alpha(x), A)$ is compact and its image under $\pi$ is all of $\Phi_{A \cdot}(Z(\alpha(x), A)$ is, for monic polynomials, the carrier space of the Banach algebra $A[x] /(\alpha(x))$; see [2] for details. If $M$ is locally constant on $Z(\alpha(x), A)$, then $Z(\alpha(x), A)$ and $\Phi_{A}$ must both decompose topologically in the way specified in

LEMMA 1.6. Suppose that $\alpha(x) \in A[x]$ is a monic polynomial. If $M$ is locally constant on $Z(\alpha(x), A)$, then there exist disjoint open sets $X_{i}, i=1,2, \cdots, s$, in $\Phi_{A}$ and disjoint open sets $Y_{i j}$ in $Z(\alpha(x), A), j=1,2, \cdots, s_{i} ; i=1,2, \cdots, s$, such that (i) $\Phi_{A}=\bigcup_{i=1}^{s} X_{i}$, (ii) $\pi^{-1}\left(X_{i}\right)=\bigcup_{j=1}^{s_{i}} Y_{i j}$, (iii) card $\pi^{-1}(h) \cap Y_{i j}$ is constant on $X_{i}$ for each $i$ and $j$ and (iv) $M$ is constant on each $Y_{i j}$.

Proof. Let $F_{j}$ denote the set $\{(h, \lambda) \in Z(\alpha(x), A): M(h, \lambda)=j\}$. Since $M$ is locally constant on $Z(\alpha(x), A)$, the $F_{j}$ 's are open subsets of $Z(\alpha(x), A)$ and hence closed since they are disjoint. Now, let $G_{i j}$ denote the set $\left\{h \in \Phi_{A}: \operatorname{card}\left(\pi^{-1}(h) \cap F_{j}\right)=i\right\}$. We will show that $G_{i j}$ is both open and closed by showing that if $h$ is in the closure $G_{i j}$ of $G_{i j}$, then $h$ is in the interior of $G_{i j}$. To this end, let $h \in \Phi_{A}$ and let $\left(h, \lambda_{k}\right)$, $k=1,2, \cdots, r(h)$, denote the points in $\pi^{-1}(h)$. We can find disjoint neighborhoods $V_{k}$ in $Z(\alpha(x), A)$ of the points $\left(h, \lambda_{k}\right)$ such that for each $k$ (i) $\pi \mid V_{k}: V_{k} \rightarrow \pi\left(V_{k}\right)$ is a homeomorphism, (ii) $M$ is constant on $V_{k}$ and (iii) $\pi\left(V_{k}\right)=\pi\left(V_{1}\right)$. Furthermore, (iv) $\pi^{-1}\left(\pi\left(V_{1}\right)\right)=\bigcup_{k=1}^{r(h)} V_{k}$. To see this, take $M$-neighborhoods $W_{k}$ of the $\left(h, \lambda_{k}\right)$ which satisfy conditions (ii) and (iv) of Proposition 1.3. In view of Lemma 1.4, we know there exists, for each $k$, a neighborhood $U_{k}$ in $Z(\alpha(x), A)$ of $\left(h, \lambda_{k}\right)$ such that $M$ is constant on $U_{k}$ and $\pi \mid U_{k}: U_{k} \rightarrow \pi\left(U_{k}\right)$ is a homeomorphism. Let $V=\bigcap_{k=1}^{r(h)} \pi\left(U_{k}\right)$. If $V_{k}$ is taken to be $\pi^{-1}(V) \cap W_{k}$, then the above conditions (i)-(iv) are satisfied.

Now, if $h \in \bar{G}_{i j}$, then there exists a $h^{\prime} \in \pi\left(V_{1}\right) \cap G_{i j}$ and hence there are distinct points $\left(h^{\prime}, \lambda_{1}^{\prime}\right), \cdots,\left(h^{\prime}, \lambda_{i}^{\prime}\right)$ in $F_{j}$. Each point $\left(h^{\prime}, \lambda_{t}\right), 1 \leqq t \leqq i$, belongs to one and only one of the $V_{k}$ 's. Denote the subscript by $k(t)$. Then $M \equiv j$ on $V_{k(t)}$. Furthermore, if $M \not \equiv j$ on $V_{k}$, then $V_{k} \cap V_{k(t)}=\varnothing, t=1,2, \cdots, i$. Thus, $\operatorname{card}\left(\pi^{-1}(h) \cap F_{j}\right)=i$ and $h$ must be in $G_{i j}$. Also, by the same argument, $\pi\left(V_{1}\right) \subset G_{i j}$ so that $G_{i j}$ is both open and closed. 
For each $h \in \Phi_{A}$, let $V(h)=\bigcap_{h \in G_{i j}} G_{i j}$. Since there are only finitely many $G_{i j}$ 's the $V(h)$ 's are both open and closed in $\Phi_{A}$. We next show that if $h^{\prime} \in V(h)$, then $V\left(h^{\prime}\right)=V(h)$. For clearly, $V\left(h^{\prime}\right) \subset V(h)$. Let $G_{i_{1}, j_{1}}, \cdots, G_{i_{n}, j . .}$ be the set of all $G_{i j}$ 's whose intersection is $V(h)$. Then $\sum_{k=1}^{n} i_{k} j_{k}=m(h)(m(\cdot)$ defined above). If the intersection $V\left(h^{\prime}\right)$ is smaller than $V(h)$, then

$$
m(h)=\sum_{k=1}^{n} i_{k} j_{k}<\sum_{\left(h^{\prime}, \lambda^{\prime}\right) \in \pi^{-1}\left(h^{\prime}\right)} M\left(h^{\prime}, \lambda^{\prime}\right)
$$

But the right-hand side is equal to $m\left(h^{\prime}\right)$. Observing that $m(\cdot)$ is constant on $\Phi_{A}$, we have a contradiction. Thus, $V(h)=V\left(h^{\prime}\right)$.

Since the sets $V(h)$ are open and either identical or disjoint, the compactness of $\Phi_{A}$ implies that there are points $h_{1}, h_{2}, \cdots, h_{s}$ in $\Phi_{A}$ such that $\Phi_{A}=\bigcup_{i=1}^{s} V\left(h_{i}\right)$ and $V\left(h_{i}\right) \cap V\left(h_{j}\right)=\varnothing$ if $i \neq j$. Let $X_{i}=V\left(h_{i}\right)$ and $Y_{i j}=\pi^{-1}\left(X_{i}\right) \cap F_{j}$. With this choice, conditions (i)-(iv) of the lemma are satisfied.

Q.E.D.

2. Factorization of polynomials. Let us recall that we are assuming that our Banach algebras are semi-simple. Examples can be given which show that this assumption is a necessary one.

Suppose that $\alpha(x), \alpha_{i}(x) \in A[x], i=1,2$, and that $\alpha(x)=\alpha_{1}(x) \alpha_{2}(x)$. Then $Z(\alpha(x), A)=Z\left(\alpha_{1}(x), A\right) \cup Z\left(\alpha_{2}(x), A\right)$. (We are assuming that not all the coefficients of $Q(x)$ vanish at the same points of $\Phi_{A}$.) Associated with each set $Z\left(\alpha_{i}(x), A\right)$ we have the multiplicity function $M_{i}$ of the polynomial $\alpha_{i}(x)$, and if we agree that $M_{i}$ is to be zero at those points of $Z(\alpha(x), A)$ for which it is not defined, then $M_{1}(h, \lambda)+M_{2}(h, \lambda)=M(h, \lambda),(h, \lambda) \in Z(\alpha(x), A)$. Furthermore, the sets $Z\left(\alpha_{i}(x), A\right)$ are closed in the prescribed topology in $Z(\alpha(x), A)$.

Suppose now that there exist closed subsets $X_{1}$ and $X_{2}$ of $Z(\alpha(x), A)$ such that $\mathrm{Z}(\alpha(x), A)=X_{1} \cup X_{2}$ and functions $M_{1}$ and $M_{2}$ which behave like multiplicity functions on $X_{1}$ and $X_{2}$, respectively, and $M_{1}+M_{2}=M$. Thus, we ask: is this enough to force a factorization over $A$ of $\alpha(x)$, with factors $\alpha_{1}(x)$ and $\alpha_{2}(x)$ such that $Z\left(\alpha_{i}(x), A\right)=X_{i}, i=1,2$. The answer is yes if $A=C(\Omega)$ but is no in general. In fact, if $A$ is regular and self-adjoint the above conditions are still not sufficient. Witness the following example. Let $A=L_{1}(I), I=$ integers, let $f \in A$ such that $|\hat{f}| \notin \hat{A}$, and let $\alpha(x)=x^{2}-g, \hat{g}=|\hat{f}|^{2}$. Then the above conditions are satisfied if $X_{i}=\left\{(h, \lambda) \in \Phi_{A} \times C: \lambda=(-1)^{i}|\hat{f}(h)|\right\}$ and $M_{i} \equiv 1, i=1,2$, but $\alpha(x)$ fails to factor into a product $\alpha_{1}(x) \alpha_{2}(x), Z\left(\alpha_{i}(x), A\right)=X_{i}, i=1,2$, since this would imply that $|\hat{f}| \in \hat{A}$. Thus, we see that the factorization of a polynomial $\alpha(x)$ over $A$ is not, in general, a topological property of the space $Z(\alpha(x), A)$. However, if $X_{1} \cap X_{2}=\varnothing$, then $\alpha(x)$ must factor as indicated above. Before proving this (Theorem 2.1) we set the following notation.

Let $\alpha(x)=\sum_{i=0}^{n} \alpha_{i} x^{i} \in A[x]$. We will use $(z)$ for $\left(z_{0}, z_{1}, \cdots, z_{n}\right),((z), \lambda)$ for $\left(z_{0}, z_{1}, \cdots, z_{n}, \lambda\right)$ and $(z(h))$ for $\left(\hat{Q}_{0}(h), \hat{Q}_{1}(h), \cdots, \hat{Q}_{n}(h)\right), h \in \Phi_{A}$. If $K$ is a compact open subset of $Z(\alpha(x), A)$ such that $\pi(K)=\Phi_{A}$, then for each $h \in \Phi_{A}$, let 
$\pi^{-1}(h) \cap K=\left\{\left(h, \lambda_{1}(h)\right), \cdots,\left(h, \lambda_{r(h)}(h)\right)\right\}$. Let $V_{i}(h) \subset K$ denote the $M$-neighborhood of $\left(h, \lambda_{i}(h)\right)$ and $U(h)$ the neighborhood in $C^{n+1}$ of $(z(h))$ which were specified in Proposition 1.3. Furthermore, let $U_{i}(h)$ denote the set

$$
\left\{((z), \lambda) \in U(h) \times C: p\left(z_{0}, z_{1}, \cdots, z_{n}, \lambda\right)=\sum_{i=0}^{n} z_{i} \lambda^{i}=0, \text { and }\left|\lambda-\lambda_{i}\right|<s_{i}\right\}
$$

(see Proposition 1.3 for the definition of $s_{i} ; s_{i}$ depends on $h$ ), $i$ varying from one to $r(h)$.

THEOREM 2.1. Let $\alpha(x)=\sum_{i=0}^{n} \alpha_{i} x^{i} \in A[x]$. If $K$ is a compact open subset of $\mathrm{Z}(\alpha(x), A)$ such that $\pi(K)=\Phi_{A}$, then there are mutually orthogonal idempotents $e_{i}$ in $A$ and polynomials $\beta_{i}(x), Q_{i}(x) \in A[x], i=1,2, \cdots, s$, such that (i) $e=\sum_{i=1}^{s} e_{i}$, (ii) $e_{i} \beta_{i}(x)$ is monic over $e_{i} A$, (iii) $K=\bigcup_{i=1}^{s} Z\left(e_{i} \beta_{i}(x), e_{i} A\right)$, and (iv) $\alpha(x)=\left(\sum_{i=1}^{s} e_{i} \beta_{i}(x)\right)\left(\sum_{i=1}^{s} e_{i} Q_{i}(x)\right)$.

Proof. For simplicity (of notation), let us first assume that the function $m(\cdot)$ (defined in the last section) is constant on $\Phi_{A}$. We use $m$ itself to denote this constant.

For each $h \in \Phi_{A}$, let $f_{j}(h)$ be defined by

$$
f_{j}(h)=(-1)^{m-j}\left(\lambda_{1}(h) \lambda_{2}(h) \cdots \lambda_{m-j}(h)+\lambda_{1}(h) \lambda_{2}(h) \cdots \lambda_{m-j-1}(h) \lambda_{m-j+1}(h)+\cdots\right)
$$

for $j=0,1, \cdots, m-1$. Clearly $f_{j} \in C\left(\Phi_{A}\right)$ for each $j$. The next step is to show that $f_{j} \in \hat{A}$.

For each $h \in \Phi_{A}$, let $F_{j, h}$ denote the function defined by

$$
F_{j, h}(z)=(-1)^{m-j}\left(\mu_{1}(z) \mu_{2}(z) \cdots \mu_{m-j}(z)+\mu_{1}(z) \mu_{2}(z) \cdots \mu_{m-j-1}(z) \mu_{m-j+1}(z)+\cdots\right)
$$

for $j=0,1, \cdots, m-1$, where $(z) \in U(h)$ and $\mu_{k}(z)$ denotes the roots of $p\left(z_{0}, z_{1}, \cdots, z_{n}, \mu\right)=0$, each repeated according to its multiplicity, such that $\left((z), \mu_{k}(z)\right) \in \bigcup_{i=1}^{r(h)} U_{i}(h)$. Each $F_{j, h}$ is analytic in $U(h)$ (cf., for example, [4]). Furthermore, if $h^{\prime} \in V(h)$, then $F_{j, h}=F_{j, h}$ in $U(h) \cap U\left(h^{\prime}\right)$, and $F_{j, h}(z(h))=f_{j}(h)$ for each $h \in \Phi_{A}$. (This says that $h \rightarrow F_{j, h}$ is a continuous mapping in the sense described in [1].) Hence by a theorem (6.2, [1]) of Arens and Calderón, $f_{j} \in \hat{A}$.

Now let $\beta_{i} \in A$ be the unique elements such that $\hat{\beta}_{j}=f_{j}$ for each $j$. If $\beta(x)$ denotes the monic polynomial $\sum_{i=0}^{m-1} \beta_{i} x^{i}+x^{m}$, then $Z(\beta(x), A)=K$ and the multiplicity function of $\beta(x)$ is precisely $M \mid K$, where $M$ is the multiplicity function of $\alpha(x)$. Since $\beta(x)$ is monic, there are polynomials $Q(x), R(x) \in A[x]$ such that $\alpha(x)=\beta(x) Q(x)+R(x)$ and the degree of $R(x)$ is less than that of $\beta(x)$. Now, for each $h \in \Phi_{A}$, we have that $\alpha_{h}(x)=\beta_{h}(x) Q_{h}(x)+R_{h}(x)$ so that $R_{h}(x)=0$ since $\beta_{h}(x)=0$ has $m$ roots (including multiplicities). Hence $R(x)=0$ and $\alpha(x)=\beta(x)$ $Q(x)$.

We now turn to the more general situation of the theorem where $m$ is not necessarily constant on $\Phi_{A}$. However, in this case $m$ is locally constant on $\Phi_{A}$ 
(cf. Lemma 1.5). Let $X_{i}, i=1,2, \cdots, s$, be the (disjoint) sets of constancy of the function $m$. By a theorem of Šilov (cf. [6]), there exist mutually perpendicular idempotents $e_{1}, e_{2}, \cdots, e_{s}$, such that $e=\sum_{i=1}^{s} e_{i}$ and $\hat{e}_{i}(h)=1$ if and only if $h \in X_{i}$. Also, $\Phi_{e_{i} A}$ is (identifiable with) $X_{i}$. Applying the first part of the proof to $e_{i} \alpha(x)$ over $e_{i} A$, for each $i$, we have polynomials $\beta_{i}(x)$ and $Q_{i}(x)$ over $A$ such that $e_{i} \alpha(x)=e_{i} \beta_{i}(x) Q_{i}(x), e_{i} \beta_{i}(x)$ monic over $e_{i} A$. Thus $\left(\sum_{i=1}^{s} e_{i} \beta_{i}(x)\right)\left(\sum_{i=1}^{s} e_{i} Q_{i}(x)\right)$ $=\sum_{i=1}^{s} e_{i} \alpha(x)=\alpha(x)$. It is clear that $Z\left(\sum_{i=1}^{s} e_{i} \beta_{i}(x), A\right)=K$. $\quad$ Q.E.D.

The factorization demonstrated above is not unique. For example, when $s=2$,

$$
\begin{aligned}
\alpha(x) & =\left(e_{1} \beta_{1}(x)+e_{2} \beta_{2}(x)\right)\left(e_{1} Q_{1}(x)+e_{2} Q_{2}(x)\right) \\
& =\left(e_{1} \beta_{1}(x)+e_{2} Q_{2}(x)\right)\left(e_{1} Q_{1}(x)+e_{2} \beta_{2}(x)\right) .
\end{aligned}
$$

We will take up this question of uniqueness in Theorem 2.4.

We now turn to the question of when a factor appears as a multiple factor If $\alpha(x), \beta(x) \in A[x]$ and if $k$ is a positive integer such that $\beta(x)^{k}=\alpha x$ ), then $M(h, \lambda) / k$ is an integer for every $(h, \lambda) \in Z(\beta(x), A)(Z(\beta(x), A)$ is a subset of $Z(\alpha(x), A))$. Without any assumptions on $\alpha(x)$, the converse is false, that is, the existence of such a $k$ need not imply that there exists a polynomial $\beta(x)$ such that $\beta(x)^{k}=\alpha(x)$. Witness the following example. Let $\Delta=\{\lambda \in C:|\lambda| \leqq 1\}$ and $A=\left\{f \in C(\Delta): f\right.$ analytic in the interior of $\Delta$ and $\left.f^{\prime}(0)=0\right\}$. If $\alpha(x)=z^{2} x^{2}-2 z^{3} x+z^{4}$, then $M$ is identically equal to two on $Z(\alpha(x), A)$ but $\alpha(x)$ is not the square of any other polynomial. This difficulty vanishes if we assume that $\alpha(x)$ is monic over $A$. That this is true follows from the corollary below, which is stated in a more general setting for later use. In the remainder of this section, we restrict our attention to monic polynomials.

In the following corollary, $Z(\alpha(x), A)$ is assumed to be the union of disjoint compact open subsets $X_{i}, i=1,2, \cdots, t$. The functions $m_{X_{i}}(\cdot)$ defined earlier will be denoted by $m_{i}(\cdot) \cdot$

Corollary 2.2. Let $\alpha(x)$ be a monic polynomial over $A$ and $X_{1}, X_{2}, \cdots, X_{t}$, be disjoint open subsets of $Z(\alpha(x), A)$, the union of which is $Z(\alpha(x), A)$. Assume that each $m_{i}(\cdot)$ is constant on $\Phi_{A}$ and denote its constant value by $m_{i}$. If there are positive integers $k_{1}, k_{2}, \cdots, k_{t}$, such that for each $i$ and every $(h, \lambda) \in X_{i}, M(h, \lambda) / k_{i}$ is an integer, then there are monic polynomials $\alpha_{i}(x), i=1,2, \cdots, t$, of degree $m_{i} / k_{i}$ over $A$ such that $Z\left(\alpha_{i}(x), A\right)=X_{i}$ and $\alpha(x)=\prod_{i=1}^{t} \alpha_{i}(x)^{k_{i}}$.

Proof. By repeated applications of the theorem, there are monic polynomials $\beta_{1}(x), \beta_{2}(x), \cdots, \beta_{t}(x) \in A[x]$ such that $\alpha(x)=\prod_{i=1}^{t} \beta_{i}(x)$ and $Z\left(\beta_{i}(x), A\right)=X_{i}$. The degree of $\beta_{i}(x)$ is $m_{i}$ and the multiplicity function of $\beta_{i}(x)$ is $M_{i}=M \mid X_{i}$. Recall that the coefficient $\beta_{i j}$ of $X_{j}$ in $\beta_{i}(x)$ satisfies

$$
\hat{\beta}_{i j}(h)=(-1)^{m_{i}-j}\left(\lambda_{1}^{(i)}(h) \cdots \lambda_{m_{i}-j}^{(i)}(h)+\cdots\right),
$$

where $\lambda_{1}^{(i)}(h), \cdots, \lambda_{m_{i}}^{(i)}(h)$ are all the roots of $\left(\beta_{i}\right)_{h}(x)=0$, each distinct root repeated 
according to its multiplicity. Let $\mu_{1}^{(i)}(h), \cdots, \mu_{n_{i}}^{(i)}(h), n_{i}=m_{i} / k_{i}$, denote all the roots of $\left(\beta_{i}\right)_{h}(x)=0$ but now each distinct $\mu_{j}^{(i)}(h)$ is to be repeated only $M\left(h, \mu_{j}^{(i)}(h)\right) / k_{i}$ times. Then the functions $\alpha_{i, j}$ defined by

$$
\alpha_{i, j}(h)=(-1)^{n_{i}-j}\left(\mu_{1}^{(i)}(h) \cdots \mu_{n_{i}-j}^{(i)}(h)+\cdots\right), \quad j=0,1,2, \cdots, n_{i}-1,
$$

are continuous on $\Phi_{A}$. Furthermore, if $\alpha_{i}(x)=\sum_{j=0}^{n_{i}-1} \alpha_{i, j} x^{i}+x^{n_{i}}$, th?n $\alpha_{i}(x)^{k_{i}}=\sum_{i=0}^{m_{i}} \hat{\beta}_{i j} x^{i}$. But this means that the coefficients of $\alpha_{i}(x)$ must lie in $\hat{A}$; for the coefficient of $x^{m_{i}-l}$ in $\alpha_{i}(x)^{k_{i}}, n_{i}=m_{i} / k_{i} \geqq l \geqq 1$, is of the form

$$
k_{i}\left(\alpha_{i n_{i}-l}\right)+\left(\text { terms in } \alpha_{i j}, n_{i}>j>n_{i}-l\right)
$$

so that by induction the coefficients of $\alpha_{i}(x)$ must lie in $\hat{A}$. Hence, $\alpha(x)=\prod_{i=1}^{t} \alpha_{i}(x)^{k_{i}}$. Since $A$ is semi-simple, we may and do take $\alpha_{i}(x)$ to be a polynomial over $A$. This proves the corollary since the $\alpha_{i}(x)$ are monic, of degree $n_{i}=m_{i} / k_{i}$ and $\mathrm{Z}\left(\alpha_{i}(x), A\right)=X_{i}$.

We now turn our attention to the case where $M$ is locally constant on $Z(\alpha(x), A)$, with $\alpha(x)$ monic.

THEOREM 2.3. Let $\alpha(x)$ be a monic polynomial over $A$. If $M$ is locally constant on $\mathrm{Z}(\alpha(x), A)$, then there are mutually orthogonal idempotents $e_{i} \in A$, positive integers $k_{i j}$ and polynomials $\alpha_{i j}(x) \in A[x], j=1,2, \cdots, s_{i} ; i=1,2, \cdots, s$, such that $e_{i} \alpha_{i j}(x)$ is monic over $e_{i} A$, the discriminant of $\prod_{j=1}^{s_{i}} e_{i} \alpha_{i j}(x)$ is inevertible in $e_{i} A$ and

$$
\alpha(x)=\sum_{i=1}^{s} e_{i} \prod_{j=1}^{s_{i}} \alpha_{i j}(x)^{k_{i j}} .
$$

Furthermore, if $\gamma(x)$ is any other polynomial over $A$ such that $\gamma_{h}(\lambda)=0$ for each $(h, \lambda) \in Z(\alpha(x), A)$, then $\gamma(x)$ is a multiple of $\beta(x)=\sum_{i=1}^{s} e_{i} \prod_{j=1}^{s_{i}} \alpha_{i j}(x)$.

Proof. Let $X_{i}, Y_{i j}, j=1,2, \cdots, s_{i} ; i=1,2, \cdots, s$ be the sets constructed in Lemma 1.6. Let $k_{i j}$ be equal to the constant value of $M$ on $Y_{i j}$. It follows fro:i Theorem 2.1 and its corollary that there are mutually orthogonal idempote: $t s$ $e_{i}, \cdots, e_{s}$ and polynomials $\alpha_{i j}(x)$ over $A$ such that $\sum_{i=1}^{s} e_{i}=e$,

$$
e_{i} \alpha(x)=\prod_{j=1}^{s_{i}} e_{i} \alpha_{i j}(x)^{k_{i j}}
$$

and $Y_{i j}=\left\{(h, \lambda) \in X_{i} \times C:\left(\alpha_{i j}\right)_{h}(\lambda)=0\right\}, i=1,2, \cdots, s$. The degree of $e_{i} \alpha_{i j}(x)$ is equal to

$$
\sum_{(h, \lambda) \in \pi^{-1}(h) \cap Y_{i j}}\left(M(h, \lambda) / k_{i j}\right)
$$

which in turn is equal to the number of points in $\pi^{-1}(h) \cap Y_{i j}, h \in X_{i}$. Hence there are as many distinct roots of $\left(\alpha_{i j}\right)_{h}(x)=0, h \in X_{i}$, as the degree of $e_{i} \alpha_{i j}(x)$ 
so that the discriminant of $e_{i} \alpha_{i j}(x)$ is invertible in $e_{i} A$. Since the $Y_{i j}$ are disjoint, the discriminant of $e_{i} \prod_{j=1}^{s_{i}} \alpha_{i j}(x)$ is invertible in $e_{i} A$.

Now suppose that $\gamma_{h}(\lambda)=0$ for all $(h, \lambda) \in Z(\alpha(x), A)$. Then $e_{i} \gamma(x)=e_{i} \beta(x) Q_{i}(x)$ $+e_{i} R_{i}(x)$ for some $Q_{i}(x)$ and $R_{i}(x)$ in $A[x]$, where the degree of $e_{i} R_{i}(x)$ is less than the degree of $e_{i} \beta(x)$ (defined above). Since $\beta_{h}(x)=0$ has no multiple roots for $h \in X_{i},\left(R_{i}\right)_{h}(x)=0$ has a greater number of distinct roots than its degree so that the coefficients of $e_{i} R_{i}(x)$ belong to every maximal ideal of $e_{i} A$. Hence $R_{i}(x)=0$ since $A$ is semi-simple and

$$
\gamma(x)=\left(\sum_{i=1}^{s} e_{i} Q_{i}(x)\right) \beta(x) .
$$

The last assertion of the above theorem says that, in other words, if $M$ is locally constant on $Z(\alpha(x), A)$, with $\alpha(x)$ monic, then the radical $A[x] /(\alpha(x))$ is a principal ideal generated by the coset $\beta(x)+(\alpha(x))$ (cf. [2]).

We return now to the question of uniqueness of the factorization. Our earlier comments showed that if $A$ is the direct sum of two of its ideals $e_{1} A$ and $e_{2} A$, $e_{i} e_{j}=\delta_{i j} e_{i}, e=e_{1}+e_{2}$, then the factorization is not unique. If $A$ is not decomposable, the factorization is still not necessarily unique. In this case $M$ is not locally constant on $Z(\alpha(x), A)$, as following theorem shows.

TheOREM 2.4. Suppose that $A$ is an indecomposable algebra. If $\alpha(x)$ is a monic polynomial over $A$ and if its multiplicity function $M$ is locally constant on $Z(\alpha(x), A)$, then a factorization of $\alpha(x)$ into irreducible factors is unique (to within units).

Proof. Recall first that $Z(\alpha(x), A)$ is compact since $\alpha(x)$ is monic. In this situation it is well known that the mapping $\pi$ from $Z(\alpha(x), A)$ onto $\Phi_{A}$ is a closed mapping.

Since $A$ is indecomposable, we know that $\Phi_{A}$ is connected (cf. [7]). Hence $Z(\alpha(x), A)$ must be the union of disjoint open connected subsets $X_{i}$ such that $\pi\left(X_{i}\right)=\Phi_{A}$. For if $Z(\alpha(x), A)=Q_{1} \cup Q_{2}$, where $Q_{1}$ and $Q_{2}$ are disjoint and open, and hence closed, then $\pi\left(Q_{i}\right)$ is both open and closed in $\Phi_{A}$ so that $\pi\left(Q_{i}\right)=\Phi_{A}$ or $Q_{i}=\varnothing$. This process of decomposition, when repeated, must terminate after a finite number of steps so that there are disjoint open connected sets $X_{1}, X_{2}, \cdots, X_{t}$ in $Z(\alpha(x), A)$ such that $\bigcup_{i=1}^{t} X_{i}=\Phi_{A}$ and $\pi\left(X_{i}\right)=\Phi_{A}$ for each $i$. Furthermore, the assumption that $M$ is locally constant on $Z(\alpha(x), A)$ implies that $M$ is constant on each $X_{i}$. This assumption together with the fact that $X_{i}$ is connected implies that the number of points in $\pi^{-1}(h) \cap X_{i}$, for each $i$, is constant on $\Phi_{A}$. Let $k_{i}$ denote the constant value of $M$ on $X_{i}$ and $n_{i}$ the card $\left(\pi^{-1}(h) \cap X_{i}\right)$. Applying Theorem 2.3, we have that there are monic polynomials $\alpha_{i}(x) \in A[x]$ such that the degree of $\alpha_{i}(x)$ is $n_{i}, \alpha(x)=\prod_{i=1}^{t} \alpha_{i}(x)^{k_{i}}$ and $Z\left(\alpha_{i}(x), A\right)=X_{i}$. Also, the discriminant of each $\alpha_{i}(x)$ is invertible in $A$. That the factors $\alpha_{i}(x)$ are irreducible follows from the argument given below.

Suppose that $\alpha(x)=\beta_{1}(x) \beta_{2}(x) \cdots \beta_{n}(x)$. We can assume without loss of gen- 
erality that $n=2$. Then $Z(\alpha(x), A)=Z\left(\beta_{1}(x), A\right) \cup Z\left(\beta_{2}(x), A\right)$ and the $Z\left(\beta_{i}(x), A\right)$ are closed in $Z(\alpha(x), A)$. We now show that each $Z\left(\beta_{i}(x), A\right)$ is also open. Let $M_{i}$ denote the function on $Z(\alpha(x), A)$ which is equal to the multiplicity function of $\beta_{i}(x)$ on $\mathrm{Z}\left(\beta_{i}(x), A\right)$ and zero otherwise. Then $M_{1}(h, \lambda)+M_{2}(h, \lambda) \equiv M(h, \lambda)$. Each point $(h, \lambda) \in Z\left(\beta_{1}(x), A\right)$ possesses a neighborhood $W_{0}$ in $Z(\alpha(x), A)$ on which $M_{1}$ is constant. To see this, choose a neighborhood $W_{0}$ in $Z(\alpha(x), A)$ of $(h, \lambda)$, on which $M$ is constant, so that $W_{0} \cap Z(\alpha(x), A)$ is a $M$-neighborhood (relative to the polynomial $\left.\beta_{1}(x)\right)$ in $Z\left(\beta_{1}(x), A\right)$ of $(h, \lambda)$ and $W_{0} \cap Z\left(\beta_{2}(x), A\right)$ (relative to the polynomial $\left.\beta_{2}(x)\right)$ is a $M$-neighborhood in $Z\left(\beta_{2}(x), A\right)$ of $(h, \lambda)$ if $(h, \lambda) \in Z\left(\beta_{2}(x), A\right)$ but $W_{0} \cap Z\left(\beta_{2}(x), A\right)=\varnothing$ if $(h, \lambda) \notin Z\left(\beta_{2}(x), A\right)$. Then $M_{i}\left(h^{\prime}, \lambda\right) \leqq M_{i}(h, \lambda)$ for each $\left(h^{\prime}, \lambda^{\prime}\right) \in W_{0}$ and $i=1,2$. If $M_{1}$ is not constant on $W_{0}$, then there is a point $\left(h^{\prime}, \lambda^{\prime}\right) \in W_{0}$ such that $M_{1}\left(h^{\prime}, \lambda^{\prime}\right)<M_{1}(h, \lambda)$. By the above restrictions on $W_{0}, M_{2}\left(h^{\prime}, \lambda^{\prime}\right) \leqq M_{2}(h, \lambda)$ so that $M\left(h^{\prime}, \lambda^{\prime}\right)=M_{1}\left(h^{\prime}, \lambda^{\prime}\right)+M_{2}\left(h^{\prime}, \lambda^{\prime}\right)$ $<M_{1}(h, \lambda)+M_{2}(h, \lambda)=M(h, \lambda)$. This is a contradiction and so it must be the case that $M_{i}$ is constant on $W_{0}$. Since $M_{1}(h, \lambda)>0, W_{0}$ is a subset of $Z\left(\beta_{1}(x), A\right)$. Thus, $Z\left(\beta_{1}(x), A\right)$ is open, as well as closed, in $Z(\alpha(x), A)$. This means that $Z\left(\beta_{1}(x), A\right)$ is the union of some of the $X_{i}$, say $X_{1}, X_{2}, \cdots, X_{s}$ (after possible relabeling) and fails to intersect the other $X_{i}$ 's. $\prod_{i=1}^{s} \alpha_{i}(x)$ is a monic polynomial with an invertible discriminant since the $Z\left(\alpha_{i}(x), A\right)$ 's are disjoint. It follows from the previous theorem that $\prod_{i=1}^{s} \alpha_{i}(x)$ is a factor of $\beta_{1}(x)$.

By continuing this process we have that

$$
\beta_{j}(x)=Q_{j} \prod_{i=1}^{t} \alpha_{i}(x)^{k_{i j}}, \quad j=1,2,
$$

for an appropriate choice of non-negative integers $k_{i j}$ and $Q_{j} \in A$. Since $Q_{1} Q_{2}=e$, the $Q_{i}$ are invertible in $A$.

Q.E.D.

3. Zeros of polynomials. In this section we deal with sufficient conditions under which $\alpha(x) \in A[x]$ has a zero in (i) a Banach algebra extension of $A$, that is, a Banach algebra $B$ in which $A$ is embedded isomorphically and isometrically, and (ii) in $A$ itself. In [2] it is shown that if $A$ is a Banach algebra and if $\beta(x) \in A[x]$ has an invertible leading coefficient, then $A[x] /(\beta(x))$ possesses a norm which makes it a Banach algebra extension of $A$, the isomorphism being the canonical one.

It is also shown in [2] that if $A$ is semi-simple and if $R$ denotes the radical of $A[x] /(\beta(x))$, then $(A[x] /(\beta(x))) / R$ possessess a norm so that it is a Banach algebra extension of $A$. Again the isomorphism is the canonical one. We now make use of these propositions in proving

THEOREM 3.1. Let $A$ be a semi-simple Banach algebra and let $\alpha(x)$ be a polynomial over $A$. If $K$ is a compact open subset of $Z(\alpha(x), A)$ such that $\pi(K)=\Phi_{A}$, then there exists a Banach algebra extension $A[b]$ of $A$ such that $\alpha(b)=0$. 
Proof. By Theorem 2.1, $\alpha(x)=\left(\sum_{i=1}^{s} e_{i} \alpha_{i}(x)\right)\left(\sum_{i=1}^{s} e_{i} Q_{i}(x)\right)$ where the $e_{i}$ are mutually orthogonal idempotents such that $e=\sum_{i=1}^{s} e_{i}$. Furthermore, the $e_{i} \alpha_{i}(x)$ are monic over $e_{i} A$ and $Z\left(\sum_{i=1}^{s} e_{i} \alpha_{i}(x), A\right)=K$. Let $m$ denote the least common multiple of the $n_{i}, n_{i}=$ degree of $e_{i} \alpha_{i}(x)$, and let $\beta(x)=\sum_{i=1}^{s} e_{i} \alpha_{i}(x)^{m / n_{i}}$. Then $\beta(x)$ is a monic polynomial over $A$ and hence has a solution in $A[x] /(\beta(x))$. Now $(A[x] /(\beta(x))) / R$ is isomorphic to $(A[x] /(\beta(x)))^{\wedge}$, the Gelfand representation of $A[x] /(\beta(x))$. Thus, $(A[x] /(\beta(x)))^{\wedge}$ is of the form $\hat{A}[b]$, where $b=(x+(\beta(x)))^{\wedge}$, and $\sum_{i=1}^{s} \hat{e}_{i} \hat{\alpha}_{i}(b)=0$. By the above comments, $(A[x] /(\beta(x))) / R$ is a Banach algebra extension of $A$; hence, $\hat{A}[b]$ is a Banach algebra extension of $A$.

Q.E.D.

Our next theorem gives a sufficient condition that $\alpha(x)=0$ has a solution in $A$, where $\alpha(x) \in A[x]$, and generalizes a theorem (in case $A$ is semi-simple, which we assume here) due to Arens and Calderón.

THEOREM 3.2. Let $A$ be a semi-simple Banach algebra. If $\alpha(x) \in A[x]$, if $f \in C\left(\Phi_{A}\right)$ such that $\hat{\alpha}(f)=0$ and if $M(\cdot, f(\cdot))$ is locally constant on $\Phi_{A}$, then $f \in \hat{A}$, that is, there is an $a \in A$ such that $\alpha(a)=0$ and $\hat{a}=f$.

Proof. Let $K$ denote the graph of $f$. Then $K$ is a compact subset of $Z(\alpha(x), A)$. By Lemma $1.4, K$ is an open subset of $Z(\alpha(x), A)$ so that by Theorem 2.1 and its corollary,

$$
\alpha(x)=\sum_{i=1}^{s} e_{i} \alpha_{i}(x)^{k_{1}} Q_{i}(x)
$$

where $K=Z\left(\sum_{i=1}^{s} e_{i} \alpha_{i}(x), A\right)$ and the degree of $e_{i} \alpha_{i}(x)$ is one. If $e_{i} \alpha_{i}(x)$ $=e_{i} x-e_{i} b_{i}$, then $\sum_{i=1}^{s}\left(e_{i} b_{i}\right)^{\wedge}$ must be $f$. Thus $f \in \hat{A}$, or equivalently, there exists $a \in A$ such that $\alpha(a)=0$ and $\hat{a}=f$.

Q.E.D.

The Arens-Calderón theorem $(4.1,[1])$ assumes that $M(\cdot, f(\cdot))$ is identically one but does not require that $A$ be semi-simple. Precisely: if $\sum_{i=0}^{n} \hat{a}_{i}(h)(f(h))^{t}=0$ and $M(h, f(h))=1$ for each $h \in \Phi_{A}$, then there exists $a \in A$ such that $\sum_{i=0}^{n} \alpha_{i} a^{i}=0$ and $\hat{a}=f$. When $M(\cdot, f(\cdot))$ is just locally constant on $\Phi_{A}$ and $A$ not semi-simple, we can not draw the stronger conclusion that there is an $a \in A$ such that $\alpha(a)=0$ and $\hat{a}=f$.

REMARK. Some of the main theorems of the Arens-Calderón paper [1] deal with functions $G\left(z_{0}, z_{1}, \cdots, z_{n}, w\right)$ which are analytic in a neighborhood of $K=\left\{\left(\hat{\alpha}_{0}(h), \hat{\alpha}_{1}(h), \cdots, \hat{\alpha}_{n}(h), f(h)\right): h \in \Phi_{A}\right\}$. Specifically, they show that if $G\left(z_{0}, z_{1}, \cdots, z_{n}, w\right)=0, G_{w}\left(z_{0}, z_{1}, \cdots, z_{n}, w\right) \neq 0$ for each $\left(z_{0}, z_{1}, \cdots, z_{n}, w\right) \in K$, then $f \in \hat{A}$ (cf. Theorem 6.8, [1]). The condition on $G_{w}$ can be replaced by the condition that the multiplicity of $f(h)$ as a root of $G\left(\hat{\alpha}_{0}(h), \hat{\alpha}_{1}(h), \cdots, \hat{\alpha}_{n}(h), \lambda\right)=0$ is locally constant on $\Phi_{A}$. If so, then $f$ once again must belong to $\hat{A}$. The proof of Theorem 3.2 can be extended so as to give a proof of the above assertion.

\section{REFERENCES}

1. R. Arens and A. P. Calderón, Analytic functions of several Banach algebra elements, Ann. of Math. (2) 6 (1955), 204-216. 
2. R. Arens and K. Hoffman, Algebraic extensions of normed algebras, Proc. Amer. Math. Soc. 7 (1956), 203-210.

3. C. Chevalley, Theory of Lie groups, Princeton Univ. Press, Princeton, N. J., 1946.

4. S. Bochner and W. T. Martin, Several complex variables, Princeton Univ. Press, Princeton, N. J., 1948.

5. W. F. Osgood, Lehrbuch der Funktionentheorie, Vol. 2 (lst part) Teubner, Leipzig, 1929.

6. C. E. Rickart, General theory of Banach algebras, Van Nostrand, New York, 1960.

7. G. E. Silov. Decomposition of a commutative normed ring Mat. Sb. (N. S) 32 (74) (1953), 353-364; Amer. Math. Soc. Transl. (2) 5 (1955), 37-48.

YALE UNIVERSITY, New Haven, Connecticut

SYRACUSE UNIVERSITY, Syracuse, New YorK 